\title{
Annual and Diurnal Wind Variations Originating from Japanese Islands
}

\author{
Masanori Onishi, Satoshi Sakai and Isao Iizawa* \\ Graduate School of Human and Environmental Studies, Kyoto University, Kyoto, Japan
}

\begin{abstract}
The convergent and divergent wind over the Japanese islands is estimated using meteorological observation data to examine the general characteristics of land and sea breeze driven by differential heating of land and sea. The results show an annual cycle wind called the "Japanese Monsoon" blows, as well as a diurnal cycle flow. These winds are caused by the temperature difference between the Japanese islands and the surrounding sea. The divergent wind field over the Japanese islands can be expressed simply as a superposition of synoptic-scale phenomena (wind fields with seasonal and daily cycles). An annual variation in the amplitude of the diurnal cycle wind is closely related to the magnitude of solar radiation.
\end{abstract}

\section{Introduction}

Land and sea breeze has been investigated by many researchers because they are closely related to the lives of people; for example, they affect the thermal environment of coastal areas and air pollution. In the Japanese islands, land and sea breeze has been studied at several locations (e.g., Yoshikado 1981 in Akita, Sendai, and Himeji regions; Mizuma 1995 in Osaka region; and Kitayama et al. 1991 in Fukuoka region). These studies, however, selected typical land and sea breeze events on fine days only, and restricted the study area to several tens of kilometers.

Fujibe (1981) analyzed land and sea breeze over the Kanto and Chubu regions. He identified that a quasi-steady wind blows seasonally as well as daily cycle wind in those areas. This study covered a wider area than the abovementioned ones did, although the areas studied were regions in Japanese islands.

These winds are driven by a temperature contrast between land and sea that commonly exists throughout the year. Therefore, the driving force of land and sea breeze should commonly exist. This is also true for the annual cycle wind over the Japanese islands, "Japanese Monsoon". Although such a seasonal wind has not yet been reported explicitly, it is natural to expect that it originates from the Japanese islands due to seasonal variation in the temperature difference between the land and surrounding sea surfaces.

In this paper, the winds over the Japanese islands are studied in a very general and simple way without any wind classification. To exclude the general wind associated with the Asian monsoon, we focus on the divergent component of the winds over the Japanese islands rather than the wind vectors at a specific observation point.

\section{The study area and meteorological data}

The Japanese islands span $2000 \mathrm{~km}$ from the northeast to southwest and are $200 \mathrm{~km}$ wide (Fig. 1). They are located off the east coast of the Asian continent. In this region, the Asian monsoon winds blow from the Pacific Ocean in summer and from the Asian continent in winter.

Corresponding author and present affiliation: Masanori Onishi, National Museum of Emerging Science and Innovation, 2-3-6 Aomi, Koto-ku, Tokyo 135-0064, Japan. E-mail: m-onishi@miraikan.jst.go.jp.

*Present affiliation: Kyoto Municipal Horikawa Senior High School. (C)2010, the Meteorological Society of Japan.
The Japanese islands are covered by 156 meteorological stations which observe hourly wind data with a $0.1 \mathrm{~m} \mathrm{~s}^{-1}$ resolution in wind speed. 57 stations of them observe solar radiation. The resolutions of the data are as follows: $22.5^{\circ}$ in wind direction (16 points of the compass), and $0.01 \mathrm{MJ} \mathrm{m}^{-2}$ in solar radiation. Of these data, selected data from appropriate stations are used in each analysis described below (Fig. 1).

\section{Divergent wind field}

To evaluate the divergent component of the wind, the Japanese islands are outlined by a folded rectangle, which is regarded as its "coastline", as shown in Fig. 1. This "coastline" consists of six sides and the length of each side is shown in Fig. 1. The divergent component of the wind velocity on each side $v_{\mathrm{i}}$ is defined perpendicular to the side and positive in the outward direction. The wind velocity $v_{\mathrm{i}}$ is obtained by averaging the data from stations within $50 \mathrm{~km}$ of $\mathrm{i}$-th "coastline". The total number of stations is 73 . The 64 stations of them are within $10 \mathrm{~km}$ from real coastline. The farthest station from sea is $33 \mathrm{~km}$ away from real coastline. The divergent wind velocity $V_{\mathrm{d}}$ is defined as

$$
V_{\mathrm{d}}=\sum_{\mathrm{i}} v_{\mathrm{i}} l_{\mathrm{i}} / \sum_{\mathrm{i}} l_{\mathrm{i}}
$$

where $l_{\mathrm{i}}$ is the length of each side of the "coastline".

The divergent wind velocity $V_{\mathrm{d}}$ is considered to represent the flow field induced by the temperature difference between land and sea surfaces, which has two dominant time scales: a day and a year. The induced velocity $V_{\mathrm{d}}$ is naturally expected to have the same time scales, such that $V_{\mathrm{d}}$ can be expressed as a function of the phase variables of two periods, as $V_{\mathrm{d}}(d, h)$, where the vari-

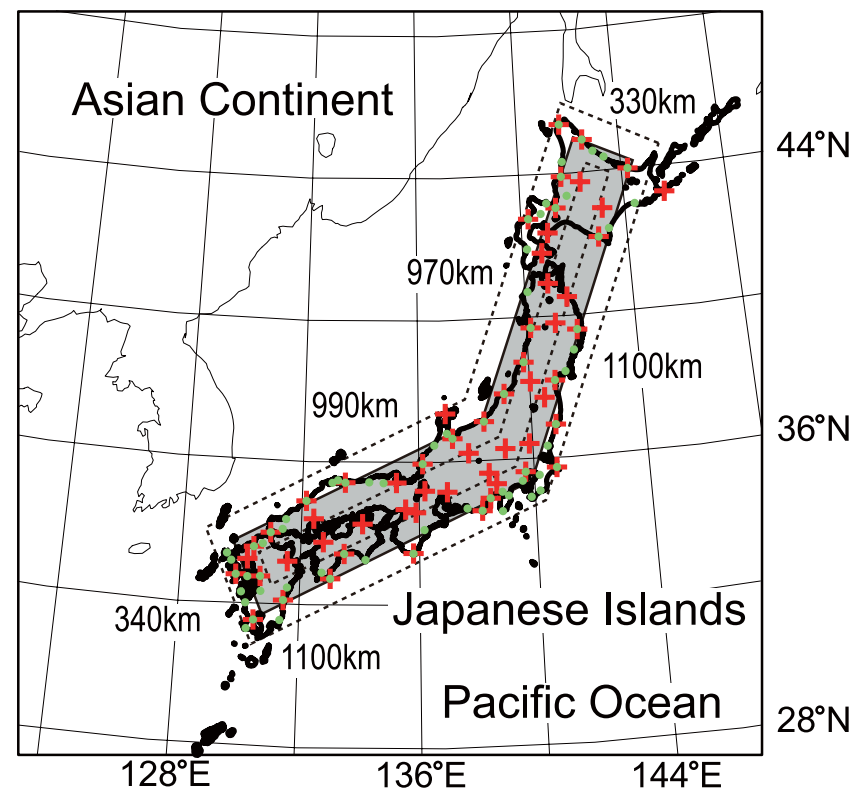

Fig. 1. Map of observation stations and a folded rectangle regarded as the "coastline." Solid line represents the "coastline" and dotted lines are drawn $50 \mathrm{~km}$ away from it. Green dots are observation stations providing wind velocity data; red crosses are those providing solar radiation data. 
ables $d$ and $h$ represent days and hours, respectively, i.e., $d=1,2$, $\ldots, 365$ and $h=1,2, \ldots, 24$. If complete periodicity is sustained, we can obtain a unique value of $V_{\mathrm{d}}$ when a certain day of the year and a certain hour of the day are assigned. To eliminate stochastic fluctuations, $V_{\mathrm{d}}$ is averaged over 10 days (from 1 to 10 , from 11 to 20 , and from 21 to last day of each month) for each hourly value and also over 10 years from 1996 to 2005 (the data on Feb. 29 is eliminated). In this paper, values averaged over 10 days are obtained by the same way.

Figure 2 shows the averaged divergent velocity $V_{\mathrm{d}}$. Annual variations are plotted along the ordinate and diurnal variations are plotted along the abscissa. Diurnal variation is observed clearly in all seasons. The value of $V_{\mathrm{d}}$ is plus during the day and the value is minus at night. In addition, a seasonal variation is also visible. In winter, the value of $V_{\mathrm{d}}$ is plus at almost all hours and in summer, the value is smaller than that in winter. Although such a seasonal wind has not been reported, Fig. 2 clearly shows a monsoon originating from the Japanese islands. The speed of this seasonal wind is comparable in magnitude to the wind speed of the diurnal variation in $V_{\mathrm{d}}$.

To examine both these winds in detail, the annual and diurnal variations are extracted from the divergent wind velocity $V_{\mathrm{d}}$. The annual variation can be obtained by averaging the daily fluctuations. Since the annual average of the divergent field is not zero, the annual variation $f(d)$ and mean divergent velocity $c$ are determined as follows. The annual variation $f(d)$ can be denoted by averaging over a diurnal cycle;

$$
f(d)=\sum_{\mathrm{h}=1}^{24} V_{\mathrm{d}}(d, h) / 24-c,
$$

where $c$ is the mean divergent velocity of $V_{\mathrm{d}}$, defined by

$$
c=\sum_{\mathrm{d}=1}^{365} \sum_{\mathrm{h}=1}^{24} V_{\mathrm{d}}(d, h) /(24 \times 365) .
$$

The annual variation $f(d)$ is plotted in Fig. 3. The peak-topeak amplitude of $f(d)$ is about $0.7 \mathrm{~m} \mathrm{~s}^{-1}$. The amplitude of the wind velocity of Asian monsoon is $2.3 \mathrm{~m} \mathrm{~s}^{-1}$ (The amplitude is estimated by averaging wind velocity data in 73 stations because the amplitude of Asian monsoon can be obtained as average wind field over Japanese islands.). The amplitude of Japanese seasonal wind is about one third of that of Asian monsoon.

The mean divergent velocity $c$ defined by Eq. (3) is obtained at a positive value of $0.16 \mathrm{~m} \mathrm{~s}^{-1}$, indicating that on an average, the wind blows out from the Japanese islands. This suggests that the annual mean temperature of the sea surface is warmer than that of the land surface due to the warm Kuroshio and Tsushima Current.

The diurnal variation $g^{\prime}(h)$ can be denoted by

$$
g^{\prime}(h)=\sum_{\mathrm{d}=1}^{365}\left\{V_{\mathrm{d}}(d, h)-f(d)\right\} / 365-c .
$$

Considering the seasonal variation in the amplitude of diurnal cycle wind, the simple diurnal variation $g^{\prime}(h)$ is normalized and the amplitude $A(d)$ is determined as follows. The normalized diurnal variation $g(h)$ is denoted by

$$
g(h)=g^{\prime}(h) /\left(\underset{\mathrm{h}^{\prime}}{\operatorname{MA} X}\left(g^{\prime}\left(h^{\prime}\right)\right)-\underset{\mathrm{h}^{\prime}}{M I N}\left(g^{\prime}\left(h^{\prime}\right)\right)\right)
$$

where $\underset{j}{\operatorname{MAX}}\left(g^{\prime}(j)\right)$ and $\underset{j}{\operatorname{MIN}}\left(g^{\prime}(j)\right)$ are functions defined by the maximum and minimum values, respectively, of $g^{\prime}(j)$ as a function of $j$. The amplitude of the diurnal variation $A(d)$ is defined as

$$
A(d)=\underset{\mathrm{h}}{\operatorname{MAX}}\left(V_{\mathrm{d}}(d, h)\right)-\operatorname{MIN}_{\mathrm{h}}\left(V_{\mathrm{d}}(d, h)\right)
$$

The diurnal variation $g(h)$ is plotted in Fig. 4. It shows asymmetric variations. In the divergent period, $g(h)$ has a sharp negative peak, while in the convergent period, it has nearly a constant value. Compared with the annual variation $f(d)$ (Fig. 3), the diur-

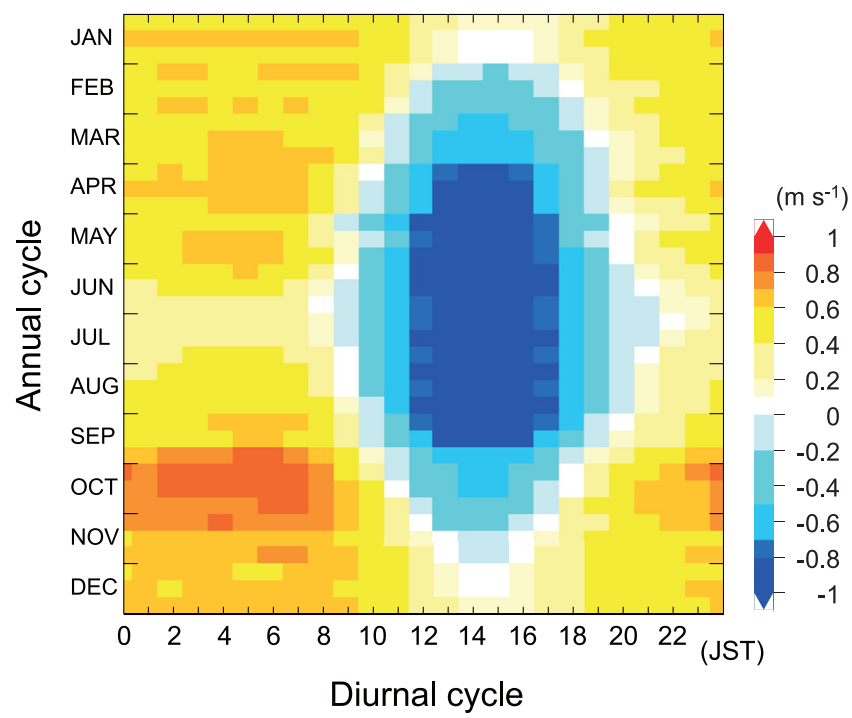

Fig. 2. Annual and diurnal cycles of divergent flow $V_{d}$. $V_{d}$ shows the average wind velocity from 1996 to 2005 . Abscissa and ordinate axes show the diurnal and annual cycles, respectively. For annual variation, $V_{\mathrm{d}}$ is averaged over 10 days.

nal variation $g(h)$ (Fig. 4) shows less fluctuation. This is because more number of data points are averaged to obtain $g(h)$ than those for $f(d)$.

The amplitude $A(d)$ of the diurnal variation is shown in Fig. 5. As expected, it usually shows large values in summer and small values in winter, but a distinct depression in July is observed. This depression can be explained by the weak solar radiation in the rainy season, as described in Section 4.2.

\section{Discussion}

\subsection{Linearity of the divergent wind field}

Although the diurnal variation $g(h)$ was calculated in the previous section by Eq. (5) using all the data obtained throughout the year, Fig. 4 also shows similar variations in the data of every season. These variations of each season are obtained by the same procedure for $g(h)$ : for each season (winter, from Dec to Feb; spring, from Mar to May; summer, from Jun to Aug; and autumn, from Sep to Nov), the mean value is subtracted from $V_{\mathrm{d}}$ and the normalized diurnal variation is obtained. These variations are similar to the original variation. This similarity suggests that the diurnal component of the wind can be expressed simply as a product of the variation $g(h)$ and its amplitude $A(d)$; in addition, it can be superimposed on the daily averaged seasonal wind $f(d)$ to reproduce the divergent wind field $V_{\mathrm{d}}$.

To verify this idea, the divergent velocity is recomposed from $c, f(d), g(h)$, and $A(d)$ as

$$
V_{\mathrm{c}}=f(d)+A(d) g(h)+c .
$$

The recomposed value $V_{\mathrm{c}}$ is shown in Fig. 6. The value is averaged over 10 days. The pattern of $V_{\mathrm{c}}$ is very similar to Fig. 2 and shows that the recomposed value $V_{\mathrm{c}}$ agrees well with the original observational value $V_{\mathrm{d}}$. It might be surprising that simple linear superposition works so well for the intricate wind system, and this result supports us to treat diurnal and annual cycle winds separately.

Figure 7 shows the correlation between the values of $V_{\mathrm{c}}$ and $V_{\mathrm{d}}$. Both values of $V_{\mathrm{c}}$ and $V_{\mathrm{d}}$ are averaged over 10 days. The black dots show the value of $V_{\mathrm{d}}$ averaged over 10 days and over 10 years, as in Fig. 6. These dots are plotted almost on a straight line with a correlation coefficient of 0.99 . The standard deviation 


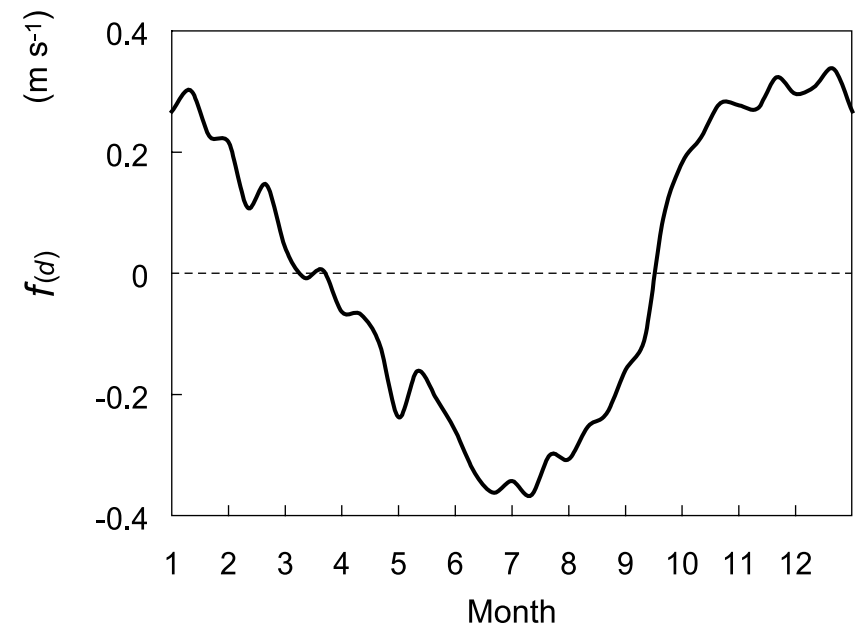

Fig. 3. Time variation of $f(d)$. The value of $f(d)$ is averaged over 10 days. Positive values indicate the divergent component.

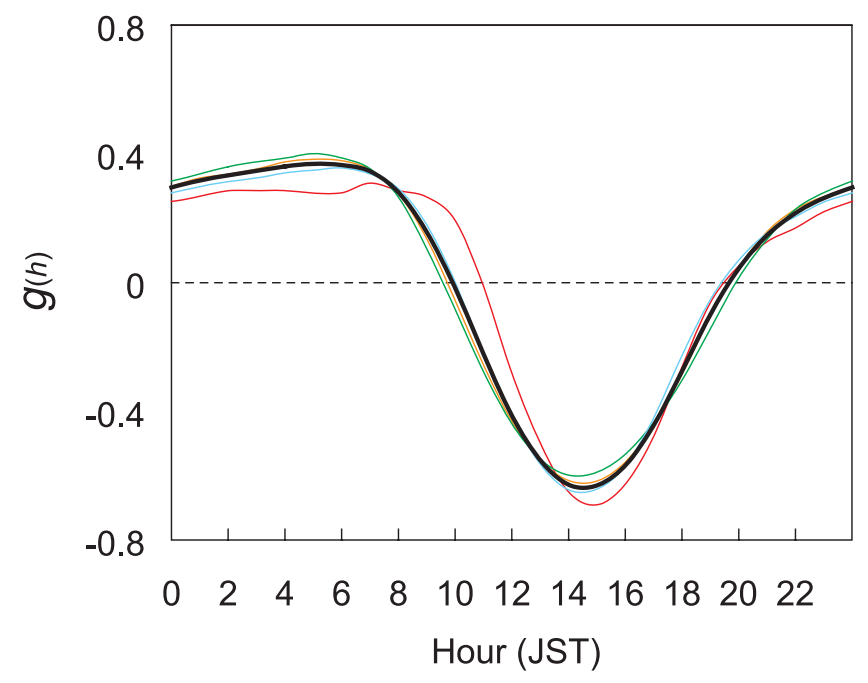

Fig. 4. Time variation of $g(h)$. Black line shows the time variation of $g(h)$. Red, orange, green, and blue lines show the time variations in winter (from Dec to Feb), spring (from Mar to May), summer (from Jun to Aug), and autumn (from Sep to Nov), respectively. Positive values indicate the divergent component.

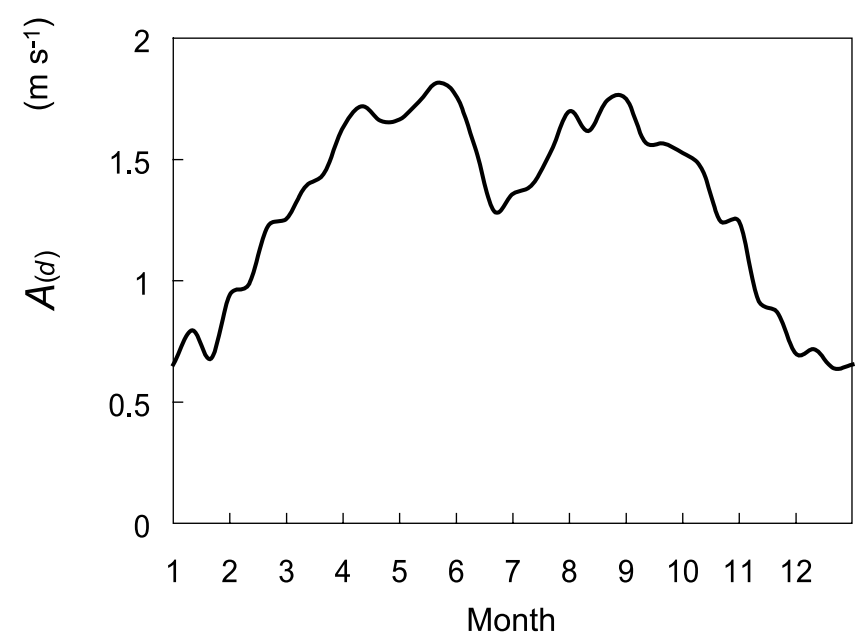

Fig. 5. Time variation of $A(d)$. The value of $A(d)$ is averaged over 10 days. of the difference between $V_{\mathrm{c}}$ and $V_{\mathrm{d}}$ is $0.054 \mathrm{~m} \mathrm{~s}^{-1}$.

Figure 7 also shows the correlation between the value of $V_{\mathrm{c}}$ and the value of $V_{\mathrm{d}}$ averaged only over 10 days each year (without the 10-year average), indicated by red dots. The standard deviation of the difference of these data is $0.18 \mathrm{~m} \mathrm{~s}^{-1}$. The ratio of the standard deviation of each year's data to that of the 10-year average data is 3.3. This indicates that the difference between $V_{\mathrm{c}}$ and $V_{\mathrm{d}}$ is caused by random events, because the ratio is almost equal to the square root of the number of averaged data $(\sqrt{10})$. These random events are supposed to be caused by synoptic-scale disturbances such as low- or high-pressure systems moving over the Japanese islands. Since the occurrence of such a disturbance on a certain day in a year is independent of the occurrences in previous years, their effects can be eliminated by simple averaging.

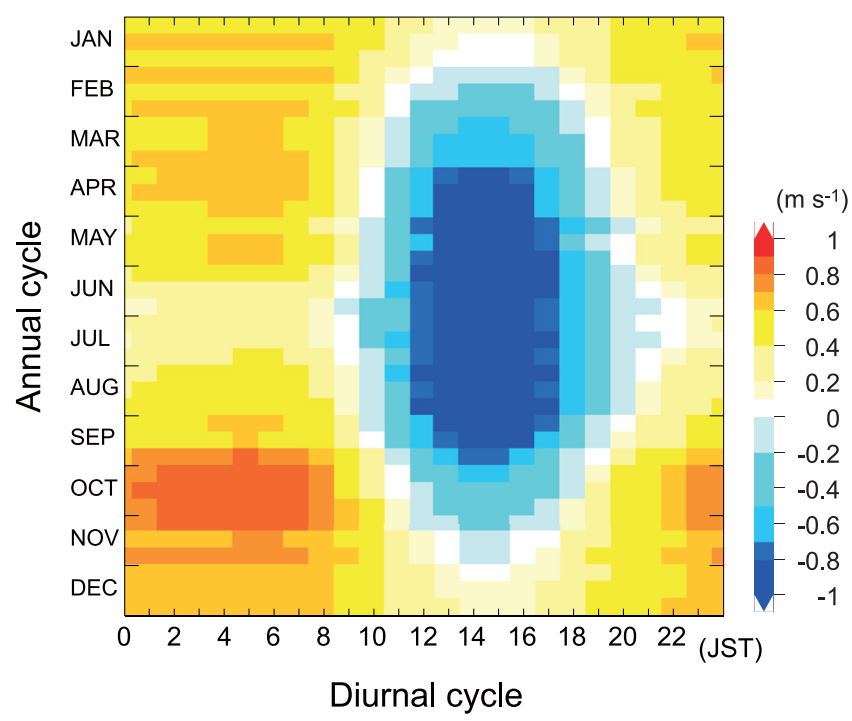

Fig. 6. Annual and diurnal cycles of $V_{c} ; V_{\mathrm{c}}=f(d)+A(d) g(h)+c$. The abscissa and ordinate axes show the diurnal and annual cycles, respectively. For annual variation, $V_{\mathrm{c}}$ is averaged over 10 days.

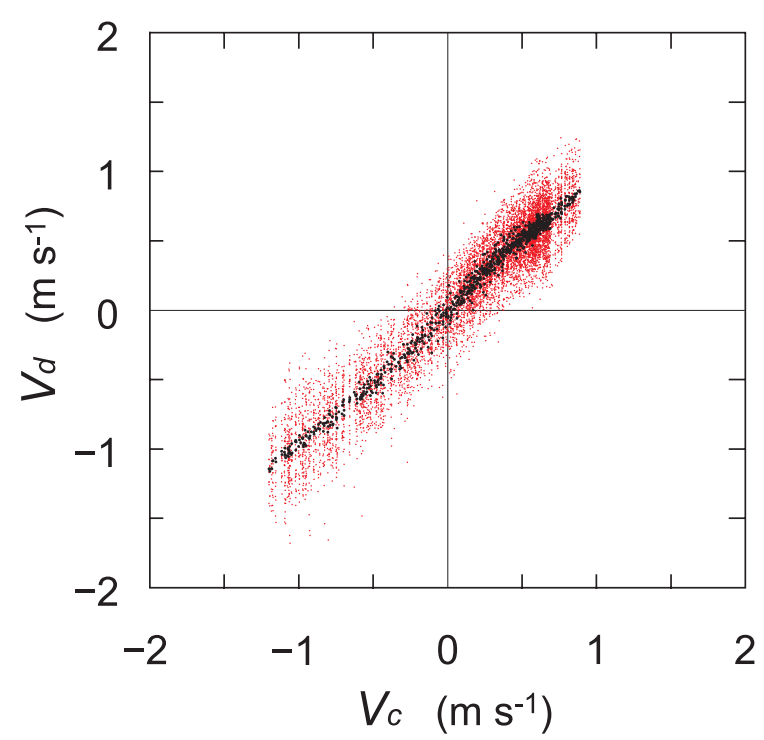

Fig. 7. Relationship between $V_{\mathrm{c}}$ and $V_{\mathrm{d}}$. Black dots show $V_{\mathrm{d}}$ averaged over 10 days and per 10 years. Red dots show $V_{\mathrm{d}}$ averaged over 10 days each year. The standard deviation of the difference between $V_{\mathrm{c}}$ and $V_{\mathrm{d}}$ averaged over 10 days and over 10 years (black dots) is $0.054 \mathrm{~m} \mathrm{~s}^{-1}$. The standard deviation of the difference between $V_{\mathrm{c}}$ and $V_{\mathrm{d}}$ averaged over 10 days each year (red dots) is $0.18 \mathrm{~m} \mathrm{~s}^{-1}$. 


\subsection{Relationship between solar radiation and divergent wind field}

Because the diurnal cycle wind is caused by the difference between the amount of solar radiation during the day and that at night, the wind velocity is supposed to be closely related to the magnitude of solar radiation itself.

The correlation between solar radiation and the amplitude of the diurnal cycle wind velocity, $A(d)$, is shown in Fig. 8. The averaged value of the solar radiation is obtained using hourly data from 57 observation stations that cover the islands almost uniformly (Fig. 1). Figure 8 shows a good correlation between the solar radiation and the diurnal wind variation. Ninomiya (1960) has reported a similar correlation between hours of sunlight and wind velocity in Niigata for 8 days in May and August 1956, when the land and sea breeze was typically dominant. The present results show that this correlation can be seen throughout the year, and not only on certain days.

Figure 8 also shows that the amplitude of the wind velocity, $1 \mathrm{~m} \mathrm{~s}^{-1}$, corresponds to the magnitude of solar radiation, $10 \mathrm{MJ}$ day $^{-1} \mathrm{~m}^{-2}$. The ratio can be roughly interpreted as follows: suppose $10 \mathrm{MJ} \mathrm{m}^{-2}$ of solar radiation is absorbed by the ground surface during the day; although we need to take temperature dependency of Bowen ratio, seasonal variation of land surface condition and so on into consideration, we assume that half of the total solar radiation, $5 \mathrm{MJ} \mathrm{m}^{-2}$, heats the atmosphere up to $1 \mathrm{~km}$ above the land. The air temperature increases by $5 \mathrm{~K}$ because the heat capacity of air is $1 \mathrm{~kJ} \mathrm{~K}^{-1} \mathrm{~m}^{-3}$. The anomaly over land can be released if the air rises by $0.5 \mathrm{~km}$, because the potential temperature generally increases at about $10 \mathrm{~K} \mathrm{~km}^{-1}$. To compensate for the upward wind, winds from the sea blow into the land. Assuming that the thickness of the wind is $1 \mathrm{~km}$, the incoming wind velocity during the day (half a day) is estimated to be $1 \mathrm{~m} \mathrm{~s}^{-1}$; the area and boundary length of the Japanese islands are assumed to be about $4 \times 10^{5} \mathrm{~km}^{2}$ and $5 \times 10^{3} \mathrm{~km}$, respectively.

To examine the relationship between solar radiation and $A(d)$ in detail, the annual variation in these values is plotted in Fig. 9. Both solar radiation and $A(d)$ are small in amplitude in July, which is the rainy season. Figure 9 shows that the amplitude of the diurnal wind variation responds to such short-term variations in solar radiation.

The annual variation in solar radiation causes the annual variation of the temperature difference between land and sea, and the variation of the temperature difference should also drive the annual cycle wind shown in Fig. 3. The amplitude of annual cycle wind $f(d)$ is less than $1 \mathrm{~m} \mathrm{~s}^{-1}$. The corresponding difference between the amount of solar radiation in summer and winter is

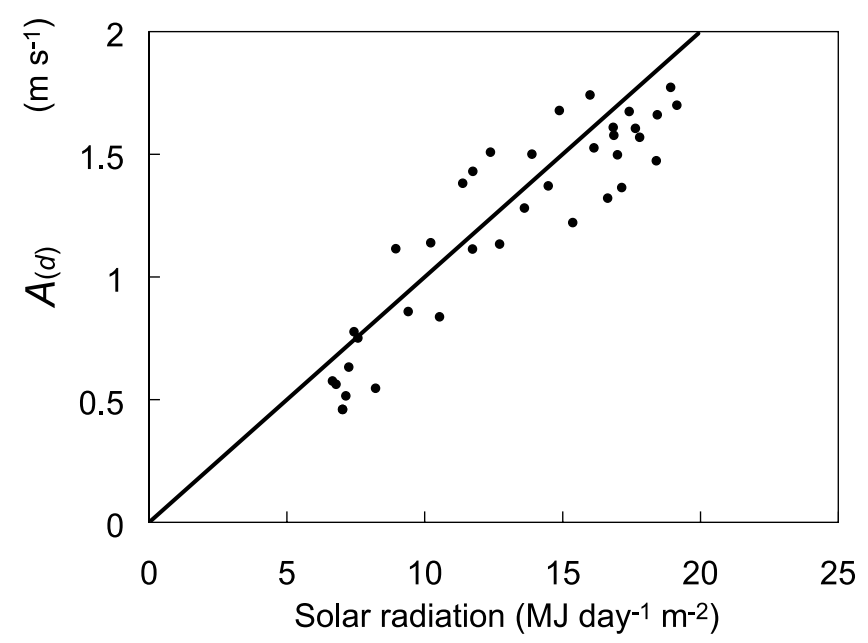

Fig. 8. Correlation between $A(d)$ and solar radiation. Black dots are observation data averaged over 10 days. Solid line shows the ratio between solar radiation and $A(d)$, assuming that solar radiation heats a $1-\mathrm{km}$ thick atmosphere, and the heat is immediately removed by advection.

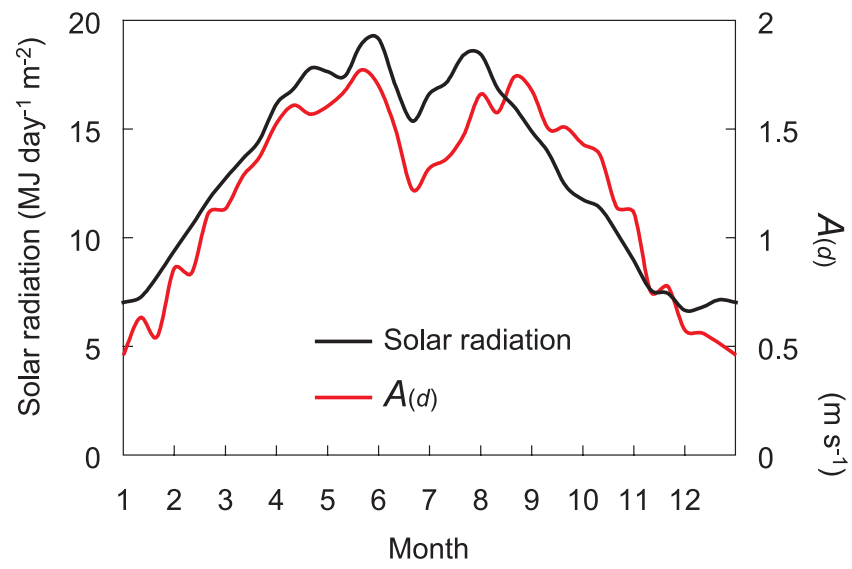

Fig. 9. Annual variation in solar radiation and $A(d)$. Black line shows solar radiation and red line shows $A(d)$. Both are averaged over 10 days. The annual variation in $A(d)$ is similar to that in solar radiation.

about $10 \mathrm{MJ}$ day $^{-1} \mathrm{~m}^{-2}$, as shown in Fig. 9. The ratio between solar radiation and wind is slightly different from that for the diurnal variation; i.e., the annual cycle wind requires a stronger variation in solar radiation than the diurnal cycle wind for the same wind speed. Also, in contrast to the diurnal variation $A(d)$, the annual variation $f(d)$ does not respond to the decrease in radiation during the rainy season.

\section{Conclusion}

The convergent and divergent wind over the Japanese islands is estimated using meteorological observation data to examine the annual and diurnal cycle winds driven by differential heating of the islands and the surrounding sea. Wind velocity data are obtained at 73 meteorological stations from 1996 to 2005. The results show the presence of the "Japanese Monsoon", which is an annual cycle wind caused by the temperature difference between the Japanese islands and the surrounding sea, as well as the diurnal cycle flow. The speed of this seasonal wind is comparable in magnitude to that of the diurnal cycle wind and the amplitude is about one third of that of Asian monsoon. Both annual and diurnal cycle wind components can be linearly superposed to reconstruct the observed wind field. An annual variation in the amplitude of the diurnal cycle wind is also shown to be closely related to the magnitude of solar radiation.

\section{References}

Fujibe, F., 1981: Seasonal characteristics of land and sea breezes. Tenki, 28, 367-375, (in Japanese).

Kitayama, H., T. Katayama, T. Hayashi, J. Tsutsumi, and A. Ishii, 1991: Statistical analysis of the sea-land breeze and its effect on the air temperature in summer. Journal of Wind Engineering and Industrial Aerodynamics, 38, 93-99.

Mizuma, M., 1995: General aspects of land and sea breezes in Osaka bay and surrounding area. J. Meteor. Soc. Japan, 73, 1029-1040.

Ninomiya, K., 1960: On the land and sea breezes in Niigata district. J. Meteor. Res., 12, 719-723, (in Japanese).

Yoshikado, H., 1981: Statistical analysis of the sea breeze pattern in relation to general weather conditions. J. Meteor. Soc. Japan, 59, 98-107.

Manuscript received 22 April 2010, accepted 16 July 2010

SOLA: http://www.jstage.jst.go.jp/browse/solal 